\title{
Contributo para um retrato dos Socorristas da Cruz Vermelha Portuguesa: Saúde, Trabalho, Coping e Suporte Social
}

(7) rpso.pt/contributo-para-um-retrato-dos-socorristas-da-cruz-vermelha-portuguesa-saude-trabalho-coping-e-suporte-social/

Oliveira A, Ferreira R, Neto F, Maia A. Contributo para um retrato dos Socorristas da Cruz Vermelha Portuguesa: Saúde, Trabalho, Coping e Suporte Social. Revista Portuguesa de Saúde Ocupacional online. 2021, volume 12, 1-12. DOI: 10.31252/RPSO.25.09.2021

\section{CONTRIBUTION TO THE PORTUGUESE RED CROSS AMBULANCE PERSONNEL PORTRAIT: HEALTH, WORK, COPING AND SOCIAL SUPPORT}

TIPO DE ESTUDO: Artigo Original

Autores: Oliveira $A(1)$, Ferreira $R(2)$, Neto $F(3)$, Maia $A(4)$.

\section{RESUMO}

\section{Introdução e objetivos}

Em Portugal, uma das entidades que opera no contexto de emergência pré-hospitalar é a Cruz Vermelha Portuguesa. Trabalhar a este nível é considerado uma atividade de risco, entre eles a exposição a níveis elevados de stresse, que pode originar o desenvolvimento de problemas de saúde. Os principais objetivos deste estudo foram descrever o perfil destes operacionais considerando variáveis de saúde, trabalho, coping e suporte social, explorar diferenças de idade/género, bem como a relação entre estas variáveis.

\section{Metodologia}

Realizou-se um estudo transversal e recorreu-se aos seguintes instrumentos: General Health Questionnaire- 28, Posttraumatic Stress Disorder Checklist 5, Warr's Job-Related Affective Well-Being Scale, Brief COPE e o Survey of Perceived Organizational Support.

\section{Resultados e Conclusões}

Os resultados mostraram níveis satisfatórios de saúde em geral e bem-estar psicológico, bons níveis de satisfação com o trabalho e níveis moderados de suporte social percebido. As estratégias de coping mais utilizadas por estes socorristas foram o coping focado na emoção. As diferenças encontradas e as relações entre variáveis identificadas constituem pistas importantes a ter em conta no planeamento e execução de ações e intervenções com estes operacionais. Otimizar as condições de trabalho dos socorristas, disponibilizar apoio e promover o desenvolvimento de competências, serão contributos cruciais para a sua saúde e bem-estar psicológico, refletindo-se na qualidade do apoio que prestam à população.

Palavras-chave: socorristas, saúde ocupacional, trabalho, coping, suporte social, psicologia do trabalho ou das organizações.

\section{ABSTRACT}

\section{Introduction and objectives}

In Portugal, one of the organizations operating in the pre-hospital emergency context is the Portuguese Red Cross. Working at this level is considered a risky activity, including exposure to high levels of stress, which may lead to the development of health problems. The main objectives of this study were describe the profile of these first responders considering health, work, coping and social support variables, explore age/gender differences, as well as the relationship between these variables.

\section{Methodology}

A cross-sectional study was conducted and the following instruments were used: General Health Questionnaire - 28, Posttraumatic Stress Disorder Checklist 5, Warr's Job-Related Affective Well-Being Scale, Brief COPE, and the Survey of Perceived Organizational Support.

\section{Results and Conclusions}

The results showed satisfactory levels of general health and psychological well-being, good levels of job satisfaction, and moderate levels of perceived social support. The coping strategies most used by these first responders were emotion-focused coping. The differences found and the relationships between the variables identified constitute important clues to be taken into account in the planning and implementation of actions and interventions with these populations. Optimizing the first responders' working conditions, providing support and promoting the development of skills will make a crucial contribution to their health and psychological well-being, which will be reflected in the quality of the support provided to the population. 
Keywords: first responders, occupational health, work, coping, social support, occupational or organizational psychology.

\section{INTRODUÇÃO}

A Cruz Vermelha Portuguesa (CVP) é uma organização humanitária pertencente ao Movimento Internacional da Cruz Vermelha e do Crescente Vermelho. A sua missão é prestar assistência humanitária e social, em especial aos mais vulneráveis, prevenindo e reparando o sofrimento e contribuindo para a defesa da vida, da saúde e da dignidade humana (1). Dentro da diversidade de equipas que compõem a estrutura operacional de emergência da CVP, estão as Equipas de Socorro e Transporte (EST), que integram os socorristas, também designados de tripulantes de ambulância. A CVP é composta por 86 estruturas locais, onde operam 245 equipas de socorro (www.cruzvermelha.pt), num total de mais de 2500 socorristas. Para desempenharem o seu papel, os socorristas podem ter dois níveis de formação: o elementar, ou seja, a formação "Tripulante de Ambulância de Transporte" (TAT) e o avançado, isto é, a formação "Tripulante de Ambulância de Socorro" (TAS).

Ao longo do tempo tem vindo a crescer o interesse pelo estudo dos profissionais que operam no contexto de emergência pré-hospitalar. Dada a imprevisibilidade, complexidade das situações a que os socorristas acorrem e outros fatores de risco ocupacional como as cargas e ruídos, é considerada uma das atividades com maiores riscos para a segurança e saúde dos seus trabalhadores (2-5). A exposição contínua a estes fatores pode torná-los mais vulneráveis ao impacto do trabalho (6), poderá influenciar as relações sociais e familiares $(7)$ e a qualidade do socorro que prestam à população $(8,9)$, sendo também importante considerar a influência de outras variáveis (como personalidade, resiliência).

\section{Saúde e bem-estar psicológico}

Quando falamos em saúde falamos em algo mais do que a ausência de doença; trata-se de um estado de completo bem-estar físico, psicológico e social (10). Especificamente, o bem-estar psicológico, refere-se a um conjunto de características como a autoaceitação, relações positivas com outros, autonomia, domínio sobre o ambiente, propósito na vida e crescimento pessoal (11).

As características inerentes ao trabalho em contexto pré-hospitalar faz com que estes profissionais estejam sujeitos a níveis de stresse mais elevados comparativamente a outros profissionais de saúde $(8,12-15)$, podendo manifestar-se num maior risco de desenvolver problemas de saúde física e mental $(4,14,16-18)$. A maior parte dos estudos aponta no sentido de as mulheres reportarem mais problemas de saúde mental em geral $(13,16,19-21)$.

\section{Trauma psicológico}

Podemos definir trauma psicológico como o impacto extremo de um acontecimento stressante no funcionamento psicológico de uma pessoa (22). Quando as reações a um acontecimento provocam mal-estar e interferem com o seu funcionamento habitual, podemos estar a falar no desenvolvimento de um quadro clínico ou perturbação, como é o caso da Perturbação de Stresse Pós-Traumático (PSPT) (23). Considerando a probabilidade de este tipo de eventos acontecerem ao longo da vida da maioria das pessoas (24) e de acordo com o número de eventos experienciados (25), podemos assumir que o risco de desenvolver trauma é maior para os profissionais que operam em emergência pré-hospitalar. Estudos apontam que cerca de $56 \%$ a $88 \%$ destes profissionais já passaram pelo menos por uma experiência potencialmente traumática $(2,26)$, apresentando um maior risco de desenvolver sintomas de PSPT $(27,28)$. A literatura tem vindo a apontar para uma maior prevalência de sintomas de PSPT nas mulheres $(29-31)$.

\section{Satisfação e bem-estar afetivo em relação ao trabalho}

A satisfação com o trabalho refere-se ao grau de satisfação com as características intrínsecas (por exemplo: método usado, variedade de tarefas, responsabilidade) e extrínsecas (condições físicas, colegas, carga horária) de um trabalho (32). O bem-estar afetivo em relação a esta variável diz respeito à avaliação que uma pessoa faz sobre o quão bem se sente no exercício da sua atividade e é influenciada por fatores pessoais e do ambiente (33). Alguns estudos verificaram níveis baixos de satisfação com o trabalho em populações semelhantes às dos socorristas $(21,34)$; no entanto, outros estudos reportaram níveis elevados $(2,35)$.

\section{Coping}

Refere-se a ações adotadas face a uma situação indutora de stresse ou, como Lazarus e Folkman (36) o definiram, conjunto de esforços para gerir as exigências internas ou externas consideradas como excedendo os recursos pessoais. Os estudos realizados com esta população têm vindo a referir que quando são ativados, e antes de chegar ao local da emergência, os socorristas antecipam cenários e procedimentos $(37,38)$, de modo a prepararem-se para a situação. Durante a prestação de socorro em situações indutoras de stresse, focam-se nos procedimentos técnicos, mantendo um certo distanciamento emocional $(38,39)$. Após os eventos, estes profissionais preferem falar com os colegas e utilizar o humor para aliviar a carga emocional das situações $(2,5,40-42)$.

\section{Suporte social percebido dos Pares e da Organização}

O suporte social tem sido amplamente reconhecido como um fator protetor para a saúde mental, especialmente em situações adversas e stressantes $(18,43,44)$. Além de permitir a expressão e partilha de emoções, especialmente as negativas, permite receber ajuda prática. Além da família e amigos, o apoio prestado pelos colegas de trabalho e pelos supervisores parece ser particularmente importante para 
os socorristas $(5,41,45-47)$. Os profissionais que se sentem apoiados e valorizados parecem experienciar níveis mais baixos de stresse negativo (48) e de sintomas de stresse traumático (49).

Em Portugal não existem estudos prévios que permitam caracterizar os socorristas da CVP. Os objetivos deste estudo foram descrever o perfil destes operacionais considerando variáveis de saúde, trabalho, coping e suporte social, bem como, explorar diferenças de género, e explorar a relação entre as variáveis em estudo com a saúde e bem-estar psicológico. Assim, os dados deste estudo serão um contributo importante no sentido de se desenvolverem ações de prevenção, promoção da saúde ocupacional, e se necessário, tratamento junto deste grupo de profissionais.

\section{METODOLOGIA}

\section{Participantes}

Este estudo integrou uma amostra de 313 socorristas da CVP, com idades entre 18 e 67 anos ( $M=33.87$; DP=9.91) e uma média de anos de experiência de $7.83(D P=6.35)$. A maioria são do género feminino $(55.9 \%)$, voluntárias/os $(67.7 \%)$, do norte do país $(59.7 \%)$, não desempenham funções de chefia (75.7\%) e têm a formação de Tripulante de Ambulância de Transporte (TAT) $(70.9 \%)$, tal como pode ser observado na tabela 1.

Instrumentos de recolha de dados

General Health Questionnaire -28 (GHQ-28) (50) versão portuguesa de Pais Ribeiro e colaboradores (51)

O GHQ é um dos instrumentos de rastreio da saúde mais utilizados, para avaliar a saúde mental ou o bem-estar psicológico (50). É composto por quatro subescalas: sintomas somáticos (GHQ-28, SS), ansiedade e insónia (GHQ-28, Al), disfunção social (GHQ-28, DS) e depressão grave (GHQ-28, DG). A resposta a cada item é dada numa escala ordinal, que varia entre 0 - 3 ("De modo nenhum" "Muito mais que o habitual"): o somatório das respostas permite obter quer o valor para cada subescala, quer um total a total. $\mathrm{O}$ valor de cada subescala varia entre $0-21$ e a nota total (GHQ-28, Total) varia entre $0-84$. Valores mais elevados indicam pior saúde mental. Uma nota total acima de 23 sugere presença de um caso para estudo.

Posttraumatic Stress Disorder Checklist 5 (PCL-5) (52); versão portuguesa de Ferreira, Ribeiro, Santos \& Maia, 2016)

A PCL-5 avalia a sintomatologia da Perturbação de Stresse Pós-Traumático (PSPT), de acordo com o DSM-5 (23). É constituída por 20 itens e quatro subescalas, que correspondem aos critérios de diagnóstico da PSPT: sintomas intrusivos (PCL-5, SI), evitamento (PCL-5, $E)$, alterações negativas na cognição e no humor (PCL-5, CH) e alterações significativas da ativação e reatividade (PCL-5, AR). As respostas são dadas numa escala de Likert de cinco pontos, que varia entre 0 - 4 ("nada" - "extremamente"). O resultado total (PCL-5 TOTAL) pode variar entre $0-80$, sendo que quanto mais elevada a pontuação, mais severa é a sintomatologia. Pode ser atribuído um diagnóstico provisório de PSPT, após identificar a existência de um evento traumático, classificando de seguida cada item, correspondente a um sintoma, com resposta $\geq 2$ como estando presente. Para além disso, alguma investigação sugere que uma pontuação de corte na PCL-5 entre 31-33 é indicativa de provável PSPT (53-55).

Warr, Cook and Wall's (1979) Job Satisfaction Scale (JSS) (56); versão portuguesa de Wilks e Neto (57)

Esta escala avalia o grau de satisfação com as caraterísticas intrínsecas e extrínsecas do trabalho (condições físicas, liberdade para escolher o modo de trabalho, colegas, reconhecimento, chefe de equipa, responsabilidade atribuída, oportunidade para usar conhecimentos e competências, relação das chefias com os membros, possibilidade de ser promovido, gestão da organização, sugestões dadas, horário, tarefas, segurança e stresse) (32). A versão original, assim como a portuguesa, contém 16 itens, todavia, neste estudo foi eliminado o item referente à satisfação com o salário, uma vez que os socorristas podem ter vínculo voluntário ou profissional (Alpha de Cronbach do instrumento com os 15 itens: .92). As respostas são dadas numa escala Likert de 1 - 5 ("Muito insatisfeito" - "Muito satisfeito"). É possível obter um índice através do somatório dos itens, onde valores mais elevados sugerem um nível de satisfação mais elevado.

Warr's (1990) Job-Related Affective Well-Being Scale (JAWS (33); versão portuguesa de Wilks e Neto (57)

A JAWS afere o bem-estar afetivo relacionado com o trabalho. É composta por 12 itens, 6 itens com emoções positivas (entusiasmado, satisfeito, otimista, confortável, interessado e relaxado) e 6 itens com emoções negativas (ansioso, melancólico, tenso, deprimido, preocupado e infeliz). As respostas são dadas numa escala Likert de 1 - 5 ("Nunca" - "Sempre"). Os itens relativos às emoções negativas são codificados de forma inversa. O resultado global obtém-se através da soma dos itens, onde valores mais elevados revelam um nível mais elevado de bem-estar afetivo no trabalho.

Brief COPE (58); versão portuguesa de Pais Ribeiro e Rodrigues (59)

O COPE avalia estilos e estratégias de coping (58). O Brief COPE é constituído por 28 itens agrupados em 14 escalas: coping ativo, planear, utilizar suporte instrumental, utilizar suporte social emocional, religião, reinterpretação positiva, auto-culpabilização, aceitação, expressão de sentimentos, negação, auto-distração, desinvestimento comportamental, uso de substâncias e humor. As respostas são dadas numa escala ordinal com quatro alternativas, que variam entre 0 - 3 ("Nunca faço isto" - "Faço sempre isto"). O instrumento pode ser ajustado, consoante o interesse dos investigadores no coping estado ou coping traço. Neste estudo optou-se pelo segundo. Apesar de originalmente o resultado final ser apresentado como um perfil, em que as subescalas não são somadas, nem há uma nota total, 
neste estudo seguiremos a subdivisão por fatores utilizada em estudos anteriores com populações similares (60-62): F1 coping focado no problema: planear, coping ativo, suporte instrumental (Alfa de Cronbach da escala neste estudo = .68); F2 coping focado na emoção: suporte emocional, aceitação, humor, reinterpretação positiva, religião (Alfa de Cronbach da escala neste estudo $=.59$ ) e F3 coping disfuncional e evitamento: negação, auto-culpabilização, desinvestimento comportamental, uso de substâncias, expressão de sentimentos (Alfa de Cronbach da escala neste estudo $=.59$ ).

Survey of Perceived Organizational Support (SPOS) (63,64); versão portuguesa de Santos e Gonçalves (65) e Suporte Percebido dos Colegas (SPC, adaptado para o presente estudo)

A SPOS avalia as crenças globais dos trabalhadores acerca do grau em que a organização valoriza os seus contributos e bem-estar (63). A versão original tem 36 itens, apesar da maioria dos estudos usar uma forma abreviada da SPOS (desde 3 a 21 itens; ex., Yoon \& Lim, 1999; Guzzo et al., 1994). De acordo com uma revisão (66), uma vez que a escala original é unidimensional e tem uma elevada fiabilidade interna, a utilização de versões mais curtas não aparenta ser problemática. Nesta investigação foram selecionados oito itens (64), com um Alfa de Cronbach = .62, correspondentes a crenças sobre bem-estar dos trabalhadores, objetivos e opiniões, favores, erros e disponibilidade da organização para ajudar o trabalhador a resolver problemas. As afirmações, iniciadas com a expressão "A/o nome da organização...", podem ser respondidas através de uma escala tipo Likert, que varia entre 1 - 7 ("Discordo totalmente" - "Concordo totalmente"). Para avaliar o suporte por parte dos colegas (PCS), adaptou-se a SPOS substituindo a palavra "A Cruz Vermelha Portuguesa" por "Os meus colegas" (Alfa de Cronbach $=.79$ ).

\section{Procedimento}

Este estudo foi aprovado pela CVP e pela Comissão de Ética da Faculdade de Psicologia e Ciências da Educação da Universidade do Porto. O pedido de colaboração foi realizado através de um email para todas as Estruturas Locais da CVP. Além da apresentação do estudo, dos seus objetivos, procedimentos e questões éticas subjacentes, reforçou-se o caráter voluntário da participação. $O$ acesso à bateria de testes, disponível numa plataforma online, Google Docs., era feito através de um link, que foi igualmente divulgado através das redes sociais, num grupo de conversação de socorristas da CVP. Previamente ao preenchimento, foi feita uma breve descrição do estudo quanto aos objetivos, ao critério de inclusão (ser socorrista no ativo), à confidencialidade e anonimato dos dados e à natureza voluntária da resposta e participação. O consentimento informado foi apresentado, sendo que os/as participantes só poderiam avançar no preenchimento do instrumento depois de assinalarem (item de preenchimento obrigatório) a sua concordância com a participação no estudo. A bateria de testes esteve disponível online entre maio de 2018 e fevereiro de 2019, tendo o processo sido concluído em março de 2019. A análise dos dados foi realizada através do IBM SPSS para Windows, versão 25.

\section{RESULTADOS}

\section{Saúde e bem-estar psicológico}

Os resultados sugerem que $30.70 \%$ dos participantes (IC 95\%: 25.6 - 36.1) apresentam alterações na saúde mental. As mulheres evidenciam mais alterações na saúde mental global $(M=21.89 ; D P=8.95)$, por comparação com os homens $(M=18.97 ; D P=9.46)$ : $t=-2.796, d f=311, p=.006, d=.32)$, bem como na escala sintomas somáticos $(t=-4.931, d f=309.748, p=.000, d=.56)$, bem como ansiedade e insónia $(t=-3.310, d f=308.748, p=.001, d=.37)$.

\section{Stresse traumático}

Dos participantes, 28.40\% ( $n=97)$ identifica exposição a um acontecimento traumático (Critério A da PSPT), com 3.8\% ( $n=12)$ acima do ponto de corte $\geq 31$ e $2.56 \%(n=8)$ cumprindo critérios para provável diagnóstico de PSPT. Os resultados da PCL-5 apresentam os seguintes valores: Pontuação Total $M=13.35$ ( $D P=14.54$ ), Sintomas Intrusivos $M=4.03$ ( $D P=4.23$ ), Evitamento $M=2.01$ ( $D P=2.38)$, Alterações nas cognições e humor $M=3.09$ ( $D P=4.52$ ) e Ativação reativa aumentada $M=3.64$ ( $D P=5.11)$. Também na sintomatologia traumática, as mulheres $(n=54)$ surgem com um resultado global mais elevado $(M=15.96 ; D P=16.25)$, por comparação com o género masculino $(n=35 ; M=9.31 ; D P=10.39): t=-2.355, d f=86.995, p=.021, d=.50$. Na subescala sintomas intrusivos, verifica-se de igual modo esta diferença $(t=-2.487, \mathrm{df}=93.780, \mathrm{p}=.015, \mathrm{~d}=.51$; tabela 2$)$.

\section{Satisfação com o trabalho}

O JSS apresenta uma pontuação total média de 53.35 ( $\mathrm{DP}=11.45)$ e a pontuação média dos itens acima do valor intermédio de 3 $(\mathrm{M}=3.56$; $\mathrm{DP}=.76)$. $\mathrm{O}$ item associado a maior satisfação é a responsabilidade atribuída $(\mathrm{M}=3.96$; $\mathrm{DP}=.96)$, e, pelo contrário, à menor satisfação a possibilidade de ser promovido ( $M=3.09$; $D P=1.10)$. Não foram identificadas diferenças de género ou idade.

\section{Bem-estar afetivo em relação ao trabalho}

O JAWS apresenta uma pontuação total média de 30.29 ( $\mathrm{DP}=9.03$ ) e a pontuação média dos itens $\mathrm{M}=2.52$ ( $\mathrm{DP}=.75)$. $\mathrm{O}$ item com a média mais elevada é o interessado $(M=3.30 ; D P=1.18)$, e, inversamente, o que tem a média mais baixa o deprimido $(M=1.88 ; D P=1.45)$. Não se observam diferenças ao nível do género ou idade.

\section{Coping}

Os fatores do Brief COPE apresentam os seguintes valores: Fator 1 - Coping Focado no Problema: M=11.39 (DP=3.28); Fator 2 Coping Focado nas Emoções: M=15.21 ( $\mathrm{DP}=4.55)$ e Fator 3 - Coping Disfuncional/Evitamento: 7.06 ( $\mathrm{DP}=3.81$ ). Não foram encontradas diferenças de género ou idade. 
O SPOS apresenta uma média de 4.18 ( $\mathrm{DP}=.94)$ e o SPC uma média de 4.57 ( $\mathrm{DP}=.87)$. O grupo etário faz variar a perceção do suporte organizacional (ANOVA one-way, $F(5,306)=2.519, p=.030$, partial $\left.\eta^{2}=.04\right)$. Se forem consideradas duas categorias de idade $(18-40$ anos e 41 ou mais anos de idade) verifica-se que o grupo com idade superior a 41 anos apresenta uma maior perceção de suporte por parte da organização ( $\mathrm{t}=-2.228, \mathrm{df}=310, \mathrm{p}=.027, \mathrm{~d}=.30$, teste $t$ student). Não se observam diferenças de género.

\section{Variáveis Cruz Vermelha Portuguesa}

As variáveis que apresentam diferenças quando comparadas caraterísticas específicas da CVP, como exercer a atividade de socorristas enquanto Voluntário versus Colaborador, ter nível de formação TAT versus TAS ou desempenhar um papel de Chefia nas Equipas de Socorro e Transporte, são as seguintes (efetuado teste $t$-student):

- Saúde e Bem-estar Psicológico: os TAT evidenciam pior saúde mental, do que os TAS ( $t=2.227, d f=309, p=.027, d=.29)$.

- Satisfação com o Trabalho: os Voluntários revelam maior satisfação, por comparação com os Colaboradores $(\mathrm{t}=5.037, \mathrm{df}=$ 157.110, $p=.000, d=.64)$ e os TAT maior do que os TAS $(t=2.713, d f=307, p=.007, d=.34)$.

- Bem-Estar Afetivo no Trabalho: os Voluntários revelam maior bem-estar, por comparação com os Colaboradores $(\mathrm{t}=2.835, \mathrm{df}=$ $311, \mathrm{p}=.005, \mathrm{~d}=.35$ ).

- Suporte Organizacional: os Voluntários sentem maior suporte organizacional, por comparação com os Colaboradores $(t=2.224$, df, 165.506, $p=.027, d=.28$ ); e os TAT maior do que os TAS ( $t=3.280, d f=146.775, p=.001, d=.42)$.

- Suporte dos Colegas: os Voluntários percebem maior suporte da parte dos colegas, por comparação com os Colaboradores $(\mathrm{t}=$ $3.861, \mathrm{df}=311, \mathrm{p}=.000, \mathrm{~d}=.45)$

Um modelo preditivo exploratório da saúde e bem-estar psicológico dos Socorristas CVP, formado pelas variáveis analisadas neste estudo, revela-se significativo: $F(11,76)=3.836, p=.000$ (Regressão Múltipla, usando o Método Enter). O modelo explica 26\% da variância (Adjusted $R^{2}=.26$ ). A tabela 2 apresenta a informação para as variáveis incluídas no modelo. Destas, o Coping Disfuncional/ Evitamento é significativo (tabela 3).

\section{DISCUSSÃO DE RESULTADOS}

A maioria dos participantes reportaram níveis satisfatórios de saúde em geral e bem-estar psicológico, resultados que contrariam a tendência de estudos anteriores $(5,18)$. No entanto, importa considerar a tendência destes profissionais em sub-reportar queixas de saúde $(67,68)$. As mulheres evidenciaram não só mais alterações ao nível da saúde global e bem-estar psicológico, bem como na escala de sintomas somáticos e na escala da ansiedade e insónia. Se, por um lado, estes dados corroboram estudos anteriores $(16,19,21,69)$, por outro lado, contrariam outros, que não identificaram diferenças entre homens e mulheres para estas variáveis (8).

Ainda que cerca de $28 \%$ dos participantes tenha estado exposto a um acontecimento potencialmente traumático, apenas cerca de $3 \%$ cumpriam critérios para um eventual diagnóstico de PSPT. Esta percentagem é bastante inferior comparativamente a outros estudos nacionais $(20,29,35,60,70)$ e internacionais $(28,71)$, com populações semelhantes. Os dados deste estudo vão ao encontro da tendência apontada por Petrie e colaboradores (28) de que as taxas de PSPT nos operacionais de emergência têm vindo a diminuir ao longo dos anos. Por outro lado, estes valores levam-nos a refletir sobre que fatores poderão estar na origem da diferença entre os socorristas da CVP e os restantes profissionais de emergência pré-hospitalar (bombeiros e técnicos do Instituto Nacional de Emergência Médica). Verificamos ainda que as mulheres socorristas exibem mais sintomas globais de PSPT e sintomas intrusivos, tal como a literatura tem vindo a apontar (30) e à semelhança de estudos prévios conduzidos com bombeiros portugueses (31) e com técnicos de emergência pré-hospitalar (29). Estes dados levam-nos a considerar a hipótese da influência de fatores biológicos e o modo como lidam e gerem as emoções associadas aos eventos $(72,73)$. No entanto, houve outros estudos que não identificaram estas diferenças nos operacionais de emergência $(8,71,74,75)$.

A média da pontuação das respostas dos participantes para a satisfação profissional revelou-se superior ao ponto intermédio da escala de resposta, indicando bons níveis de satisfação com o trabalho, tal como sugerem outros estudos anteriores (2,76). No que diz respeito ao bem-estar afetivo em relação ao trabalho, o valor da média das respostas dos participantes revelou-se próximo do ponto intermédio da escala de resposta, sugerindo um nível moderado de bem-estar em relação ao trabalho. Uma das razões que pode estar relacionada com estes níveis de satisfação e bem-estar afetivo é o facto de o trabalho no contexto de emergência ser um trabalho pessoalmente gratificante e recompensador $(46,77,78)$.

Quanto ao coping, os dados revelaram que os socorristas da CVP utilizam mais estratégias de coping focado nas emoções, dentro das quais se encontram o suporte emocional, a aceitação e o humor. Estes dados estão de acordo com outros estudos anteriores (62), mas contrariam outros realizados com populações similares, em que as estratégias de coping focado no problema são as mais utilizadas $(60,61)$. Algumas estratégias focadas nas emoções, como é o caso do humor, podem ter um efeito positivo, uma vez que possibilita um distanciamento emocional entre os profissionais e a situação, promovem um sentido de mestria a lidar com as exigências do seu trabalho e promovem a coesão grupal, criando um "código de grupo" $(2,5,8,40,41,79-81)$. Além disso, o facto de estes profissionais refletirem e conversarem sobre a situação e o seu desempenho com os seus colegas de tripulação e amigos $(5,37,38,79)$, é uma oportunidade de reinterpretação positiva, crescimento pessoal e melhoria profissional (44). O coping adaptativo está associado a níveis mais baixos de stresse, PSPT e a melhor bem-estar psicológico $(62,79,82)$, revelando um impacto positivo na gestão dos incidentes e no processo de adaptação após um evento traumático $(83,84)$. As estratégias de coping focado no problema mostram-se apropriadas quando o stressor é controlável, ao passo que as focadas na emoção são mais indicadas quando o stressor parece ser incontrolável (85). Os resultados apontam para o facto das estratégias de coping disfuncional ou evitamento serem as menos utilizadas, corroborando a maioria dos estudos anteriores (86-88). Comparativamente aos homens, as mulheres parecem utilizar mais estratégias de coping 
focado no problema e de coping disfuncional/evitamento, à semelhança de outros estudos prévios $(60,87)$. Se por um lado o coping focado no problema pode ter um efeito positivo, por outro, o coping disfuncional/evitamento podem favorecer sintomas intrusivos, de ativação emocional e de evitamento (40), e se utilizadas isoladamente ou de forma inflexível, podem tornar-se prejudiciais $(89,90)$.

A análise dos dados sugere que o coping disfuncional/evitamento é uma variável que está correlacionada com a saúde e bem-estar psicológico. Também outros estudos prévios identificaram o valor preditivo desta dimensão em termos de problemas psicológicos, como é o caso do burnout e da fadiga de compaixão (87). Além disso, quando associado a baixos níveis de suporte social, este tipo de coping prediz sintomatologia de PSPT (91). Não obstante, estudos recentes têm vindo a verificar que este tipo de coping pode ser benéfico em algumas situações críticas e na recuperação após os eventos $(82,92)$. Exemplos destas situações poderão ser aquelas em que os socorristas têm que manter o seu foco nos protocolos e procedimentos de socorro. Será importante, em estudos futuros, considerar a variável "tempo decorrido desde o evento", de forma a perceber a influência desta variável no sucesso das estratégias. Estes dados realçam a importância de sensibilizar os socorristas para ações que os ajude a lidar não só com os desafios do dia-a-dia, como também com os eventos críticos com que se deparam. Ações de sensibilização, formação e treino são por isso ações de extrema relevância e que devem ser desenvolvidas continuamente.

Relativamente ao suporte percebido por parte da Cruz Vermelha, os resultados parecem indicar níveis moderados, quer por parte da instituição, quer por parte dos colegas. A literatura é uníssona ao afirmar que ter o suporte por parte dos outros e trabalhar num ambiente de apoio tem um impacto positivo na saúde mental e no bem-estar destes profissionais (93-95) e na facilitação de respostas positivas após eventos potencialmente traumáticos $(2,13,18,87,96-98)$. Os socorristas mais velhos apresentam uma maior perceção de suporte por parte da organização, o que pode estar relacionado com a visão que têm da própria instituição. Considerando o vínculo com a CVP, os socorristas voluntários sentem maior suporte organizacional e maior suporte por parte dos colegas. Estes dados poderão refletir o valor que o voluntariado - um dos princípios fundamentais da CVP- representa e a consequente atenção e eventual apoio dados aos voluntários, que representam a maioria dos socorristas da CVP. Percebendo o impacto que o apoio tem no desempenho dos seus papéis (99), torna-se essencial desenvolver ações que garantam a manutenção deste apoio. Também os socorristas com formação TAT reportam sentir maior suporte por parte da organização. Verificamos que a maioria dos socorristas com este nível de formação são voluntários, pelo que na origem desta perceção poderá estar a valorização dada ao trabalho voluntário desempenhado por estes socorristas. Considerando o efeito protetor que o suporte social parece assumir para estes profissionais (5), os resultados deste estudo sugerem que este apoio percebido poderá ajudar a reduzir os sentimentos decorrentes da sobrecarga de trabalho e do stresse ocupacional $(9,100)$. Nesse sentido, torna-se essencial garantir o apoio, quer seja através de medidas e ações promovidas pela organização, quer seja o apoio prestado pelos colegas.

\section{CONCLUSÃO}

Os socorristas da CVP reportaram níveis satisfatórios de saúde em geral e bem-estar psicológico, bons níveis de satisfação com o trabalho e níveis moderados de suporte percebido por parte da CVP. Relativamente às estratégias de coping, os resultados revelaram que os socorristas da CVP usam mais estratégias focadas na emoção. As diferenças encontradas e as variáveis correlacionadas identificadas para as várias dimensões dão-nos pistas importantes a considerar aquando do planeamento e execução de ações e intervenções com estes profissionais. A saúde e o bem-estar destes profissionais interfere não só com a dimensão individual, mas também ao nível familiar, organizacional e comunitário, pelo que a sua monitorização contínua deverá ser uma das prioridades por parte das estruturas onde estes profissionais operam. A otimização das condições de trabalho dos socorristas e a orientação para práticas promotoras de saúde será um importante contributo para o seu bem-estar psicológico e para a qualidade do apoio que prestam à população. Dada a impossibilidade de controlar a exposição a eventos com potencial traumático, importa capacitar estes operacionais para o uso de estratégias de coping adaptativas para lidar com os desafios do dia-a-dia. A preparação destes profissionais para lidar com este tipo de eventos, através da formação e treino é fundamental para a sua saúde e bem-estar. O apoio deverá ser uma componente basilar das estruturas de emergência numa lógica de continuidade. Por um lado, através da promoção de uma cultura de apoio e da disponibilização de um sistema de apoio estruturado (como sistema de apoio de pares); por outro lado, encorajando a utilização da rede de suporte informal (86).

Não obstante a originalidade e pertinência do presente estudo, existem algumas limitações que levam a que as inferências sejam feitas com cautela. Desde logo, a existência do viés da desejabilidade social associado aos estudos de autorrelato. Seria importante que estudos futuros pudessem contemplar a dimensão qualitativa do fenómeno e variáveis em estudo, de modo a fornecer uma visão mais holística e aprofundada desta realidade. Apesar das respostas adversas e sintomas psicopatológicos em profissionais de atividades de risco, a literatura tem vindo a apontar para as experiências e trajetórias positivas que esta atividade oferece $(46,104,105)$. Nesse sentido, seria importante em estudos futuros considerar variáveis como são exemplos a resiliência, o engagement e o crescimento póstraumático. Neste estudo foi avaliado o suporte percebido por parte da organização e por parte dos colegas. Seria importante em estudos futuros avaliar outras fontes de suporte informal, nomeadamente, o suporte por parte da família e de amigos. Estes dados foram recolhidos num período prévio à pandemia COVID-19, pelo que seria importante compreender estas dimensões à luz da atualidade.

Este estudo representa um contributo importante para o conhecimento desta classe profissional e lança pistas, quer para a Cruz Vermelha Portuguesa quer para outras entidades que operem no contexto de emergência pré-hospitalar, no que diz respeito ao desenvolvimento de ações de prevenção, promoção e, se necessário, intervenção no âmbito da saúde ocupacional. Simultaneamente, pode ser um bom ponto de partida no âmbito da literacia em saúde mental, quer para os socorristas individualmente, quer para os profissionais de saúde mental e as entidades que deles também têm de cuidar.

\section{QUESTÕES ÉTICAS E LEGAIS}

Foram asseguradas todas as questões éticas e legais necessárias para a prossecução deste estudo. 


\section{CONFLITOS DE INTERESSE}

Os autores declaram não haver quaisquer conflitos de interesse.

\section{BIBLIOGRAFIA}

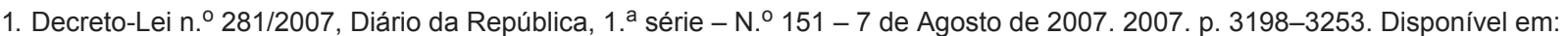
https://dre.pt/application/conteudo/637403

2. Alexander D, Klein S. Ambulance personnel and critical incidents. Br J Psychiatry. 2001;178(1):76-81.DOI: 10.1192/bjp.178.1.76

3. Bährer-Kohler S. (ed.). Burnout for Experts - Prevention in the Context of Living and Working. Switzerland: Springer; 2013. Available from: https://www.springer.com/gp/book/9781461443902

4. Benedek D, Fullerton C, Ursano R. First Responders: Mental Health Consequences of Natural and Human-Made Disasters for Public Health and Public Safety Workers. Annu Rev Public Health. 2007 Apr;28(1):55-68. doi:

10.1146/annurev.publhealth.28.021406.144037

5. Regehr C, Goldberg G, Hughes J. Exposure to human tragedy, empathy, and trauma in ambulance paramedics. Am J Orthopsychiatry. 2002; 72(4):505-13. DOI:10.1037//0002-9432.72.4.505

6. Maia A. Factores Preditores de PTSD e Critérios de Selecção em Profissionais de Actuação na Crise. In: Psiquiatria da Catástrofe. 2007. p. 263-76. Disponível em: http://repositorium.sdum.uminho.pt/bitstream/1822/7790/1/FACTORES\%20PREDITORES\%20DE\%20PTSD\%20\%20e\%20crit\%c3\%a9r

7. Regehr C, Leblanc V. Stress and trauma in the emergency services. Langon-Fox, Janice, Cooper CL. (ed.). Handbook of stress in the occupations. UK: Edward Elgar Publishing Limited; 2011. p. 201-17.

8. Bennett P, Williams Y, Page N, Hood K, Woollard M, Vetter N. Associations between organizational and incident factors and emotional distress in emergency ambulance personnel. Br J Clin Psychol. 2005;44(Pt 2):215-26. Available from: http://www.ncbi.nlm.nih.gov/pubmed/16004656

9. Kleim B, Westphal M. Mental health in first responders: A review and recommendation for prevention and intervention strategies. Traumatology (Tallahass Fla). 2011;17(4):17-24. Available from: http://tmt.sagepub.com/content/17/4/17.full.pdf+html

10. WHO. Constitution of WHO - Basic Documents. Vol. Supplement, The World Health Organization (WHO). 2006. p. 1-157. Available from: https://www.yumpu.com/en/document/read/7282878/who-constitution-world-health-organization

11. Ryff $C D$, Singer BH. Know thyself and become what you are: A eudaimonic approach to psychological well-being. J Happiness Stud. 2008;9(1):13-39. DOI: 10.1007/s10902-006-9019-0

12. Sterud T, Ekeberg O, Hem E. Health status in the ambulance services: a systematic review. BMC Health Serv Res. 2006;6(1):82. DOI.10.1186/1472-6963-6-82

13. Sterud T, Hem E, Ekeberg $\varnothing$, Lau B. Occupational stressors and its organizational and individual correlates: A nationwide study of Norwegian ambulance personnel. BMC Emerg Med. 2008;8:1-11. Available from: https://bmcemergmed.biomedcentral.com/articles/10.1186/1471-227X-8-16

14. Gallagher S, Mcgilloway S. Living in critical times: The impact of critical incidents on frontline ambulance personnel - a qualitative perspective. Int J Emerg Ment Health. 2007 Summer;9(3):215-23. Available from:https://pubmed.ncbi.nlm.nih.gov/18372663/

15. Plat MJ, Frings-Dresen MHW, Sluiter JK. A systematic review of job-specific workers' health surveillance activities for fire-fighting, ambulance, police and military personnel. Int Arch Occup Environ Health. 2011;84(8):839-57. DOI: 10.1007/s00420-011-0614-y

16. Aasa U, Brulin C, Angquist K, Barnekow-Bergkvist M. Work-related psychosocial factors, worry about work conditions and health complaints among female and male ambulance personnel. Scand J Caring Sci. 2005 Sep;19(3):251-8. DOI: 10.1111/j.14716712.2005.00333.x

17. Jones S. Describing the Mental Health Profile of First Responders: A Systematic Review. J Am Psychiatr Nurses Assoc. 2017;23(3):200-14. DOI: $10.1177 / 1078390317695266$

18. Van der Ploeg E, Kleber R. Acute and chronic job stressors among ambulance personnel: predictors of health symptoms. Occup Environ Med. 2003 Jun 1;60(Suppl I):40i - 46. DOI:10.1136/oem.60.supp__1.i40

19. Jahnke S, Poston W, Haddock C, Murphy B. Firefighting and mental health: Experiences of repeated exposure to trauma. Work. 2016 Apr 15;53(4):737-44. DOI:10.3233/WOR-162255

20. Marcelino D, Figueiras M, Claudino A. Impacto da exposição a incidentes críticos na saúde e bem-estar psicológico dos tripulantes de ambulância. Psicol Saúde Doenças. 2012;13(1):110-6. Disponível em: https://www.redalyc.org/pdf/362/36224324010.pdf

21. Sterud T, Hem E, Lau B, Ekeberg O. A comparison of general and ambulance specific stressors: predictors of job satisfaction and health problems in a nationwide one-year follow-up study of Norwegian ambulance personnel. J Occup Med Toxicol. 2011;6(1):10. DOI: $10.1186 / 1745-6673-6-10$

22. Guerreiro D, Brito B, Baptista JL, Galvão F. Stresse pós-traumático: Os mecanismos do trauma. Acta Med Port. 2007;20(4):347-54. Disponível em: https://www.actamedicaportuguesa.com/revista/index.php/amp/article/viewFile/866/540

23. American Psychiatric Association. Diagnostic and Statistical Manual of Mental Disorders. Fifth Edition. Arlington, VA: American Psychiatric Association, 2013

24. Kessler R, Aguilar-Gaxiola S, Alonso J, Benjet C, Bromet E, Cardoso G et al. Trauma and PTSD in the WHO World Mental Health Surveys. Eur J Psychotraumatol. 2017;8. DOI: 10.1080/20008198.2017.1353383

25. Vogt D, King D, King L. Risk pathways for PTSD: Making sense of the literature. Handb PTSD Sci Pract. 2014;(November):146165*. Available from: https://psycnet.apa.org/record/2007-14029-006

26. Del Ben K, Scotti J, Chen Y, Fortson B. Prevalence of posttraumatic stress disorder symptoms in firefighters. Work Stress. 2006;20(1):37-48. DOI: 10.1080/02678370600679512

27. Berger W, Coutinho E, Figueira I, Marques-Portella C, Luz M, Neylan T et al. Rescuers at risk: a systematic review and metaregression analysis of the worldwide current prevalence and correlates of PTSD in rescue workers. Soc Psychiatry Psychiatr Epidemiol. 2012;47(6):1001-11. DOI: 10.1007/s00127-011-0408-2 
28. Petrie K, Milligan-Saville J, Gayed A, Deady M, Phelps A, Dell L et al. Prevalence of PTSD and common mental disorders amongst ambulance personnel: a systematic review and meta-analysis. Soc Psychiatry Psychiatr Epidemiol. 2018;0(0):1-13. DOI: 10.1007/s00127-018-1539-5

29. Cunha S, Queirós C, Fonseca S, Campos R. Resiliência como Preditor do Impacto Traumático em Técnicos de Emergência PréHospitalar. Int J Work Cond. 13 (2017): 51-67. Available from: https://repositorio-aberto.up.pt/bitstream/10216/106526/2/205901.pdf

30. Olff M. Sex and gender differences in post-traumatic stress disorder: an update. Eur J Psychotraumatol. 2017;8(sup4):1351204. DOI:10.1080/20008198.2017.1351204

31. Fernandes F, Pinheiro R. Avaliação da Perturbação de Stresse Pós-Traumático nos Bombeiros Voluntários Portugueses. Rev Técnica e Form - Esc Nac Bombeiros. 2004;(32):7-19.

32. Warr P, Cook J, Wall T. Scales for the measurement of some work attitudes and aspects of psychological well-being. J Occup Psychol. 1979 Jun;52(2):129-48. DOI: 10.1111/j.2044-8325.1979.tb00448.x

33. Warr P. The measurement of well-being and other aspects of mental health. J Occup Psychol. 1990 Sep;63(3):193-210. DOI: 10.1111/j.2044-8325.1990.tb00521.x

34. San I. The Factors Affecting Job Satisfaction of Emergency Medical Services Professionals in Ankara. Cyprus J Med Sci. 2019;4(3):177-82. DOI: 10.5152/cjms.2019.848

35. Miguel V, Vara N, Queirós C. Satisfação com o trabalho como preditor do burnout em bombeiros assalariados. Int J Work Cond. 2014;(8). Disponível em: https://repositorio-aberto.up.pt/bitstream/10216/77620/2/96270.pdf

36. Lazarus R, Folkman S. Stress, apprasail and coping.pdf. New York: Springer Publishing Company; 1984. p. 445.

37. Avraham N, Goldblatt H, Yafe E. Paramedics' experiences and coping strategies when encountering critical incidents. Qual Health Res. 2014;24(2):194-208.DOI: 10.1177/1049732313519867

38. Oliveira A, Neto F, Teixeira F, Maia Â. Working in prehospital emergency contexts: Stress, coping and support from the perspective of ambulance personnel. Int J Work Heal Manag. 2019;12(6):469-82. DOI:10.1108/IJWHM-01-2019-0004

39. Smith A., Roberts K. Interventions for post-traumatic stress disorder and psychological distress in emergency ambulance personnel: a review of the literature. Emerg Med J. 2003;20(1):75-8. DOI:10.1136/emj.20.1.75

40. Essex B, Scott L. Chronic Stress andAssociated Coping Strategies Among Volunteer EMS Personnel. Prehospital Emerg Care. 2008;12(1):69-75. DOI:10.1080/10903120701707955

41. Halpern J, Gurevich M, Schwartz B, Brazeau P. Interventions for critical incident stress in emergency medical services: A qualitative study. Stress Heal. 2009;25(2):139-49. DOI:10.1002/smi.1230

42. Regehr C, Millar D. Situation critical: High demand, low control, and low support in paramedic organizations. Traumatology (Tallahass Fla) [Internet]. 2007;13(1):49-58. DOI:10.1177/1534765607299912

43. Brooks S, Dunn R, Amlôt R, Greenberg N, Rubin G. Social and occupational factors associated with psychological distress and disorder among disaster responders : a systematic review. BMC Psychol. 2016;4(18):1-13. DOI: 10.1186/s40359-016-0120-9

44. Mildenhall J. Occupational stress, paramedic informal coping strategies: a review of the literature. J Paramed Pract. 2012;4(6):31828. DOI: $10.12968 / j p a r .2012 .4 .6 .318$

45. Grauwiler P, Barocas B, Mills L. Police peer support programs: Current knowledge and practice. Int J Emerg Ment Health. 2008;10(1):27-38. Available from: https://www.ncbi.nlm.nih.gov/pubmed/18546757

46. Shakespeare-Finch J, Smith S, Gow K, Embelton G, Baird L. The prevalence of Post-Traumatic Growth in Emergency Ambulance Personnel. Traumatology (Tallahass Fla). 2003;9(1):58-71. DOI:10.1177/153476560300900104

47. Prati G, Pietrantoni $L$. The relation of perceived and received social support to mental health among first responders: A metaanalytic review. J Community Psychol. 2010 Apr;38(3):403-17. DOI:10.1002/jcop.20371

48. Kleim B, Westphal M. Mental Health in First Responders: A Review and Recommendation for Prevention and Intervention Strategies. Traumatology (Tallahass Fla). 2011;17(4):17-24. DOI: 10.1177/1534765611429079

49. Regehr C, Hill J, Knott T, Sault B. Social support, self-efficacy and trauma in new recruits and experienced firefighters. Stress Heal. 2003;19(4):189-93.DOI: 10.1002/smi.974

50. Goldberg D, Hillier V. A scaled version of the General Health Questionnaire. Psychol Med. 1979 Feb 9;9(1):139-45. DOI: $10.1017 / \mathrm{s} 0033291700021644$

51. Pais Ribeiro J, Silva M, Abrantes C, Coelho M, Nunes J. Ulterior validação do questionário de saúde geral de goldberg de 28 itens. Psicol Saúde Doenças. 2015;16(3):278-85. Disponível em:

https://www.researchgate.net/publication/317470058_Ulterior_validacao_do_questionario_de_saude_geral_de_goldberg_de_28_itens

52. Weathers F, Litz B, Keane T, Palmieri P, Marx B, Schnurr P. The PTSD Checklist for DSM-5 (PCL-5). 2013. Available from: www.ptsd.va.gov.

53. Blevins C, Weathers F, Davis M, Witte T, Domino J. The Posttraumatic Stress Disorder Checklist for DSM-5 (PCL-5): Development and initial psychometric evaluation. J Trauma Stress. 2015;28:489-98. DOI: 10.1002/jts.22059

54. Bovin M, Marx B, Weathers F, Gallagher M, Rodriguez P, Schnurr P, Keane T. Psychometric properties of the PTSD Checklist for Diagnostic and Statistical Manual of Mental Disorders-Fifth Edition (PCL-5) in Veterans. Psychol Assess. 2015;28:1379-91.DOI: 10.1037/pas0000254

55. Wortmann J, Jordan A, Weathers F, Resick P, Dondanville K, Hall-Clark B et al. Psychometric analysis of the PTSD Checklist-5 (PCL-5) among treatment-seeking military service members. Psychol Assess. 2016;28:1392-403. DOI: 10.1037/pas0000260

56. Warr P, Cook J, Wall T. Scales for the measurement of some work attitudes and aspects of psychological well-being. J Occup Psychol. 1979;52(2):129-48. DOI: 10.1111/j.2044-8325.1979.tb00448.x

57. Wilks D, Neto F. Workplace Well-being, Gender and Age: Examining the "Double Jeopardy” Effect. Soc Indic Res. 2013;114(3):87590. DOI: $10.1007 / \mathrm{s} 11205-012-0177-7$

58. Carver C, Scheier M, Weintraub K. Assessing Coping Strategies: A Theoretically Based Approach. J Pers Soc Psychol. 1989;56(2):267-83. DOI: 10.1037//0022-3514.56.2.267

59. Ribeiro J, Rodrigues A. Questões acerca do coping: a propósito do estudo de adaptação do brief cope. Psicol Saúde Doenças. 2004;V(1):3-15. Disponível em: https://repositorio-aberto.up.pt/bitstream/10216/5515/2/83746.pdf 
60. Fonseca S, Cunha S, Campos R, Goncalves S, Queiros C. Occupational Health of Pre-Hospital Emergency Technicians: The Contribution of Trauma and Coping. Int J Work Cond. 2019;(17):69-88. Available from:

https://ricot.com.pt/artigos/1/IJWC.17_Fonseca.et.al._p.69.88.pdf

61. Kerai S, Khan U, Islam M, Asad N, Razzak J, Pasha O. Post-traumatic stress disorder and its predictors in emergency medical service personnel: a cross-sectional study from Karachi, Pakistan. BMC Emerg Med. 2017;17(1):26. DOI: 10.1186/s12873-0170140-7

62. Jamal Y. Coping Strategies as a Mediator of Hardiness and Stress among Rescue Workers. Stud Ethno-Medicine. 2017;11(3):2018. DOI: $10.1080 / 09735070.2017 .1356033$

63. Eisenberger R, Huntington R, Hutchison S, Sowa D. Perceived organizational support. J Appl Psychol. 1986 Aug;71(3):500-7. Available from: http://doi.apa.org/getdoi.cfm?doi=10.1037/0021-9010.71.3.500

64. Eisenberger R, Stinglhamber F, Vandenberghe C, Sucharski I, Rhoades L. Perceived supervisor support: Contributions to perceived organizational support and employee retention. J Appl Psychol. 2002;87(3):565-73. DOI: 10.1037/0021-9010.87.3.565

65. Santos J, Gonçalves G. Contribuição para a adaptação portuguesa da escala de Percepção de Suporte Organizacional de Eisenberger, Huntington, Hutchison e Sowa (1986). Laboratório Psicol. 2010;8(2). DOI: 10.14417/lp.642

66. Rhoades L, Eisenberger R. Perceived organizational support: A review of the literature. J Appl Psychol. 2002;87(4):698-714. DOI: 10.1037/0021-9010.87.4.698

67. Bakker A, Gaillard A, Van Veldhoven M, Hertogs R. The Impact of Critical Incidents on Mental Health: An Exploratory Pilot Study into the Moderating Effects of Social Support on the Impact of Adverse Events in Dutch Rescue Workers. Policing. 2015;1-11. DOI: 10.1093/police/pav038

68. Griffin E. I Get By With a Little Help From My Friends: Peer Emotional Support in the Emergency Department. J Emerg Nurs. 2008 Dec;34(6):547-9. DOI: 10.1016/j:jen.2008.09.006

69. Marcelino D, Figueiras M. Symptomatology related to trauma after the expressive writing technique: an exploratory study with Portuguese firefighters. Psychol Community Heal. 2012;1(1):95-107. Available from: http://pch.psychopen.eu/article/view/11

70. Pinto R, Henriques S, Jongenelen I, Carvalho C, Maia A. The Strongest Correlates of PTSD for Firefighters: Number, Recency, Frequency, or Perceived Threat of Traumatic Events? J Trauma Stress. 2015;28(5):434-40. DOI: 10.1002/jts.22035

71. Berger W, Silva E, Coutinho F, Figueira I, Portella C, Luz M et al. Rescuers at risk: a systematic review and meta regression analysis of the worldwide current prevalence and correlates of PTSD in rescue workers. 2012;47(6). DOI: 10.1007/s00127-0110408-2

72. Ramikie T, Ressler K. Mechanisms of Sex Differences in Fear and Posttraumatic Stress Disorder. Biol Psychiatry. 2018 May;83(10):876-85. DOI: 10.1016/j.biopsych.2017.11.016

73. Christiansen D, Hansen M. Accounting for sex differences in PTSD: A multi-variable mediation model. Eur J Psychotraumatol. 2015 Dec 19;6(1):26068. DOI: 10.3402/ejpt.v6.26068

74. Maia A, Ribeiro E. The psychological impact of motor vehicle accidents on emergency service workers. Eur J Emerg Med. 2010;17(5):296-301. DOI: 10.1097/mej.0b013e3283356213

75. Marcelino D, Figueiras M. A perturbação pós-stress traumático nos socorristas de emergência pré-hospitalar: influência do sentido interno de coerência e da personalidade. Psicol Saúde Doenças. 2007;8(1):95-108. Disponível em: https://www.redalyc.org/pdf/362/36280107.pdf

76. Vara N, Queirós C. Burnout - Um risco no desempenho e satisfação profissional nos bombeiros que trabalham na emergência préhospitalar. Territorium. 2009;16:173-8. DOI: 10.14195/1647-7723_16_17

77. Hetherington A. Counselling skills in the context of the emergency services. In: Hetherington A, editor. The use of counselling skills in the Emergency Services. Philadelphia: Open University Press; 2001. p. 1-10.

78. McAllister M, McKinnon J. The importance of teaching and learning resilience in the health disciplines: A critical review of the literature. Nurse Educ Today. 2009;29(4):371-9. DOI: 10.1016/j.nedt.2008.10.011

79. Jonsson A, Segesten K, Mattsson B. Post-traumatic stress among Swedish ambulance personnel. Emerg Med J. 2003;20(1):79-84. Available from: https://emj.bmj.com/content/20/1/79.short

80. Scott T. Expression of humour by emergency personnel involved in sudden deathwork. Mortality. 2007;12(4):350-64. DOI: $10.1080 / 13576270701609766$

81. Rowe A, Regehr C. Whatever gets you through today: An examination of cynical humor among emergency service professionals. J Loss Trauma. 2010;15(5):448-64. DOI: 10.1080/15325024.2010.507661

82. Arble E, Daugherty A, Arnetz B. Models of first responder coping: Police officers as a unique population. Stress Heal. 2018;34(5):612-21. DOI: 10.1002/smi.2821

83. LeBlanc V, Regehr C, Birze A, King K, Scott A, MacDonald R et al. The Association Between Posttraumatic Stress, Coping, and Acute Stress Responses in Paramedics. Traumatology (Tallahass Fla). 2011;17(4):10-6. DOI: 10.1177/1534765611429078

84. Calhoun L, Tedeschi R. The Foundations of Posttraumatic Growth: An Expanded Framework. In: Calhoun LG, Tedeschi RG, editors. Handbook of posttraumatic growth: Research \& practice. New York: Lawrence Erlbaum Associates Publishers; 2006. p. 3-23.

85. Park C, Armeli S, Tennen H. Appraisal-Coping Goodness of Fit: A Daily Internet Study. Personal Soc Psychol Bull. 2004 May 2;30(5):558-69. DOI: 10.1177/0146167203262855

86. Boland L, Mink P, Kamrud J, Jeruzal J, Stevens A. Social Support Outside the Workplace, Coping Styles, and Burnout in a Cohort of EMS Providers From Minnesota. Work Heal Saf. 2019;67(8):414-22. DOI: 10.1177/2165079919829154

87. Prati G, Pietrantoni L. Optimism, Social Support, and Coping Strategies As Factors Contributing to Posttraumatic Growth: A Meta Analysis. J Loss Trauma Int Perspect Stress Coping. 2009;14(5):364-88. DOI: 10.1080/15325020902724271

88. Vara N, Queirós C, Kaiseler M. Estratégias de coping e emoções como preditoras do risco de burnout em bombeiros. In: Lourenço LF Mateus MA, editors. Riscos: naturais, antropomórficos e mistos, homenagem ao Professor Doutor Fernando Rebelo. Coimbra: Departamento de Geografia da Faculdade de Letras, Universidade de Coimbra. 2013; p. 585-598

89. Anshel M. A Conceptual Model and Implications for Coping with Stressful Events in Police Work. Crim Justice Behav. 2000 Jun 30;27(3):375-400. DOI: 10.1177/0093854800027003006

90. Littleton H, Horsley S, John S, Nelson D V. Trauma coping strategies and psychological distress: A meta-analysis. J Trauma Stress. 2007 Dec;20(6):977-88. DOI: 10.1002/jts.20276 
91. Meyer E, Zimering R, Daly E, Knight J, Kamholz B, Gulliver S. Predictors of posttraumatic stress disorder and other psychological symptoms in trauma-exposed firefighters. Psychol Serv. 2012 Feb;9(1):1-15. DOI: 10.1037/a0026414

92. Flannery R. Treating Psychological Trauma in First Responders: A Multi-Modal Paradigm. Psychiatr Q. 2015;86(2):261-7. DOI: 10.1007/s11126-014-9329-z

93. Prati G, Pietrantoni L, Cicognani E. Coping strategies and collective efficacy as mediators between stress appraisal and quality of life among rescue workers. Sport Exerc Perform Psychol. 2011;1(S):84-93. DOI: 10.1037/a0021298

94. Setti I, Lourel M, Argentero P. The role of affective commitment and perceived social support in protecting emergency workers against burnout and vicarious traumatization. Traumatology (Tallahass Fla). 2016;22(4):261-70. DOI: 10.1037/trm0000072

95. Shakespeare-Finch J, Rees A, Armstrong D. Social Support, Self-efficacy, Trauma and Well-Being in Emergency Medical Dispatchers. Soc Indic Res. 2015;123(2):549-65. DOI: 10.1007/s11205-014-0749-9

96. Oginska-Bulik N. Social support and negative and positive outcomes of experienced traumatic events in a group of male emergency service workers. Int J Occup Saf Ergon. 2015;21(2):119-27. DOI: 10.1080/10803548.2015.1028232

97. Shakespeare-Finch J, Daley E. Workplace Belongingness, Distress, and Resilience in Emergency Service Workers. Psychol Trauma Theory, Res Pract Policy. 2016;1-4. DOI: 10.1037/tra0000108

98. Zadow AJ, Dollard MF, Mclinton SS, Lawrence P, Tuckey MR. Psychosocial safety climate, emotional exhaustion, and work injuries in healthcare workplaces. Stress Heal. 2017 Dec;33(5):558-69. DOI: 10.1002/smi.2740

99. Setti I, Zito M, Colombo L, Cortese C, Ghislieri C, Argentero P. Well-Being and affective commitment among ambulance volunteers: A mediational model of Job Burnout. J Soc Serv Res. 2018;44(2):236-48.DOI: 10.1080/01488376.2018.1442898

100. Bowling N, Alarcon G, Bragg C, Hartman M. A meta-analytic examination of the potential correlates and consequences of workload. Work Stress. 2015 Apr 3;29(2):95-113. DOI: 10.1080/02678373.2015.1033037

101. Shakespeare-Finch J. Primary and Secondary Trauma in Emergency Personnel. Traumatology (Tallahass Fla). 2011;17(4):1-2. DOI: $10.1177 / 1534765611431834$

102. Petrie K, Gayed A, Bryan B, Deady M, Madan I, Savic A et al. The importance of manager support for the mental health and wellbeing of ambulance personnel. PLoS One. 2018;13(5). DOI: 10.1371/journal.pone.0197802

103. Betlehem J, Horvath A, Jeges S, Gondocs Z, Nemeth T, Kukla A, et al. How Healthy Are Ambulance Personnel in Central Europe? Eval Heal Prof. 2014;37(3):394-406. DOI: 10.1177/0163278712472501

104. Bonanno G. Resilience in the face of loss and potential trauma. Curr Dir Psychol Sci. 2005;14(3):135-8. DOI: 10.1002/jckp.20283

105. Brooks S, Amlôt R, Rubin G, Greenberg N. Psychological resilience and post-traumatic growth in disaster-exposed organisations: Overview of the literature. J R Army Med Corps. 2018;1-5. DOI: 10.1136/jramc-2017-000876

Tabela 1. Caracterização da amostra

\begin{tabular}{|c|c|c|c|}
\hline & & $N=313$ & $\%$ \\
\hline \multirow[t]{2}{*}{ Género } & Masculino & 138 & 44.10 \\
\hline & Feminino & 175 & 55.90 \\
\hline \multirow{2}{*}{$\begin{array}{l}\text { Idade } \\
M=33.87 ; D P=9.91\end{array}$} & $18-20$ anos & 11 & 3.50 \\
\hline & $21-30$ anos & 122 & 39.0 \\
\hline \multirow{4}{*}{$\operatorname{Min}=18 ; \operatorname{Max}=67$} & $31-40$ anos & 101 & 32.3 \\
\hline & $41-50$ anos & 60 & 19.2 \\
\hline & $51-60$ anos & 17 & 5.40 \\
\hline & +60 anos & 2 & 0.60 \\
\hline \multirow{3}{*}{ Escolaridade } & Básico & 44 & 14.06 \\
\hline & Secundário & 128 & 40.89 \\
\hline & Superior & 141 & 45.05 \\
\hline \multirow{5}{*}{ Zona do país } & $\begin{array}{l}\text { Norte } \\
\text { Centro }\end{array}$ & $\begin{array}{l}187 \\
61\end{array}$ & $\begin{array}{l}59.70 \\
19.50\end{array}$ \\
\hline & Lisboa e Tejo & 34 & 10.90 \\
\hline & Alentejo & 18 & 5.80 \\
\hline & Algarve & 9 & 2.90 \\
\hline & Madeira & 4 & 1.30 \\
\hline Vínculo à Cruz Vermelha & $\begin{array}{l}\text { Voluntário } \\
\text { Profissional }\end{array}$ & $\begin{array}{l}212 \\
101\end{array}$ & $\begin{array}{l}67.70 \\
43.30\end{array}$ \\
\hline \multirow{2}{*}{$\begin{array}{l}\text { Anos de Experiência } \\
M=7.83 ; \mathrm{DP}=6.35\end{array}$} & $0-5$ anos & 144 & 46.0 \\
\hline & $6-10$ anos & 82 & 26.2 \\
\hline Min= 0; Max=35 & $11-15$ anos & 53 & 16.9 \\
\hline
\end{tabular}




\begin{tabular}{llll}
\cline { 2 - 4 } & \multicolumn{1}{c}{$16-20$ anos } & 21 & 6.70 \\
\cline { 2 - 4 } & $21-25$ anos & 9 & 2.90 \\
\cline { 2 - 4 } & +25 anos & 4 & 1.30 \\
\hline Nível de formação & TAT & 222 & 70.90 \\
& TAS & 89 & 28.40 \\
\hline Papel de chefia & Sim & 76 & 24.30 \\
& Não & 237 & 75.70
\end{tabular}

Tabela 2. Diferenças de Género: Saúde e bem-estar psicológico e Stresse traumático

\begin{tabular}{|c|c|c|c|c|c|c|c|c|}
\hline & \multicolumn{2}{|c|}{ Masculino } & \multicolumn{2}{|c|}{ Feminino } & \multirow[t]{2}{*}{$d f$} & \multirow[t]{2}{*}{$t$} & \multirow[t]{2}{*}{$p$} & \multirow[t]{2}{*}{$d$} \\
\hline & M & DP & M & DP & & & & \\
\hline GHQ-28 SS & 3.82 & 2.95 & 5.61 & 3.47 & 309.748 & -4.931 & $.000^{* *}$ & .56 \\
\hline GHQ-28 Al & 3.88 & 4.08 & 5.53 & 4.75 & 308.748 & -3.310 & $.001^{*}$ & .37 \\
\hline GHQ-28 DS & 10.09 & 4.21 & 9.49 & 3.36 & 257.900 & 1.371 & .172 & .16 \\
\hline GHQ-28 DG & 1.18 & 2.41 & 1.26 & 2.06 & 311 & -.300 & .764 & .04 \\
\hline GHQ-28 TOTAL & 18.97 & 9.46 & 21.89 & 8.95 & 311 & 2.796 & $.006^{*}$ & .32 \\
\hline PCL-5 SI & 2.82 & 3.30 & 4.81 & 4.60 & 93.780 & -2.487 & $.015^{\star}$ & .51 \\
\hline PCL-5 E & 1.51 & 1.98 & 2.33 & 2.57 & 89.600 & -1.736 & .086 & .36 \\
\hline PCL-5 CH & 2.32 & 3.20 & 3.58 & 5.15 & 94 & -1.326 & .145 & .30 \\
\hline PCL-5 AR & 2.70 & 3.90 & 4.27 & 5.74 & 90 & -1.453 & .122 & .33 \\
\hline PCL-5 TOTAL & 9.31 & 10.39 & 15.96 & 16.25 & 86.995 & -2.355 & $.021^{*}$ & .50 \\
\hline
\end{tabular}

Nota. ${ }^{*} p<.050 .{ }^{* *} p<.001$

Tabela 3. Regressão múltipla para as variáveis correlacionadas com a saúde e bem-estar psicológico

\begin{tabular}{llllll} 
Variável & $B$ & IC $95 \%$ & $\beta$ & $t$ & $p$ \\
\hline Sexo & .57 & {$[-3.21,4.35]$} & .03 & .30 & .766 \\
\hline Formação & -3.78 & {$[-7.92, .35]$} & -.21 & -1.82 & .072 \\
\hline Anos de Experiência & .09 & {$[-.21, .40]$} & .07 & .62 & .541 \\
\hline PSPT & .06 & {$[-.07, .20]$} & .10 & .91 & .364 \\
\hline Satisfação com o Trabalho & -.78 & {$[-3.59,2.04]$} & -.06 & -.55 & .584 \\
\hline Bem-Estar Afetivo no Trabalho & -1.11 & {$[-3.69,1.47]$} & -.10 & -.86 & .395 \\
\hline Suporte Organização & -1.53 & {$[-3.98, .93]$} & -.15 & -1.24 & .218 \\
\hline Suporte Colegas & -.10 & {$[-.28, .33]$} & -.08 & -.60 & .550 \\
\hline Coping Focado no Problema & -.14 & {$[-.91, .64]$} & -.05 & -.35 & .726 \\
\hline Coping Focado nas Emoções & -.27 & {$[-.26, .80]$} & .14 & 1.03 & .307 \\
\hline Coping Disfuncional / Evitamento & -.85 & {$[.33,1.36]$} & .38 & 3.27. & $.002^{*}$
\end{tabular}

Nota. $n=83$. Adjusted $R^{2}=.26$. IC $=$ intervalo de confiança para $B .{ }^{*} p<.050$ 
Doutoranda em Psicologia na Faculdade de Psicologia e Ciências da Educação da Universidade do Porto. Especialista na área da Psicologia da Justiça e da Psicotraumatologia. Tem experiência na intervenção em situações de crise e catástrofe e no voluntariado de cariz humanitário a nível nacional e internacional. É voluntária na Cruz Vermelha Portuguesa desde 2009 e coordena uma Equipa de Saúde Mental e Apoio Psicossocial. Trabalha na Associação Portuguesa de Apoio à Vítima (APAV) enquanto gestora do Gabinete de Apoio à Vítima no Departamento de Investigação e Ação Penal (DIAP) da Comarca de Faro e é responsável pela Unidade de Apoio à Vitimação em Massa. Morada correspondência dos Leitores: Rua Antero de Quental, 9, $2^{\circ}$ piso, Gabinete 12, 8000-210 Faro. E-MAIL: anaoliveirapsicologa@gmail.com. ORCID: https://orcid.org/0000-0002-4171-1971

\section{(2) Randdy Ferreira}

É psicólogo da Cruz Vermelha Portuguesa. Ao longo do tempo, especializou-se na área da Psicotraumatologia, com um percurso formativo que inclui países como EUA, Israel ou Dinamarca. No âmbito da Saúde Mental e Apoio Psicossocial, já participou em missões/ operações nacionais e internacionais (incêndios florestais 2017 Portugal ou Ciclone Idai 2019 em Moçambique). Colabora ainda com organizações como a ONU ou a UE. 4430-107 Vila Nova de Gaia. E-MAIL: randdy.ferreira@cvpgaia.org

\section{(3)Félix Fernando Monteiro Neto}

É Professor Catedrático Emérito da Universidade do Porto. Os seus interesses de investigação incluem psicologia intercultural e psicologia social (em particular, migrações, bem-estar subjetivo, amor, solidão e perdão)- Autor de 19 livros e de mais de 400 artigos científicos. 4200-135 Porto. E-MAIL: fneto@fpce.up.pt. ORCID: https://orcid.org/0000-0003-0112-880X

\section{(4)Ângela Costa Maia}

É vice-presidente da Escola de Psicologia e coordena o laboratório "vítimas, ofensores e sistema de justiça" no Centro de Investigação em Psicologia, Universidade do Minho. Interessa-se por compreender os fatores associados ao impacto da exposição à adversidade e trauma e os percursos da vitimização à delinquência; tendo tido financiamento dos seus projetos por vários organismos governamentais. Nos últimos anos integrou a direção da International Society of Behavioral Medicine e é membro do Conselho Científico do Centro de Recursos de Stress do Ministério da Defesa. 4710-057 Braga. E-MAIL: angelam@psi.uminho.pt. ORCID: https://orcid.org/0000-00031343-9699 